\title{
A Novel Method for Performance Analysis of Compartmentalized Reservoirs
}

\author{
Mohammad Sadeq Shahamat ${ }^{1 *}$, Hamidreza Hamdi ${ }^{2}$, Lovis Mattar ${ }^{1}$ and Roberto Aguilera ${ }^{2}$ \\ ${ }^{1}$ IHS Canada, Sun Life Plaza, East Tower Suite 800, 112 -4th Avenue S.W., Calgary, AB, T2P OH3, Calgary - Canada \\ 2 University of Calgary, 2500 University Dr. NW, Calgary, AB, T2N 1N4, Calgary - Canada \\ e-mail: sadeq.shahamat@ihs.com \\ * Corresponding author
}

\begin{abstract}
This paper presents a simple analytical model for performance analysis of compartmentalized reservoirs producing under Constant Terminal Rate (CTR) and Constant Terminal Pressure (CTP). The model is based on the well-known material balance and boundary dominated flow equations and is written in terms of capacitance and resistance of a production and a support compartment. These capacitance and resistance terms account for a combination of reservoir parameters which enable the developed model to be used for characterizing such systems. In addition to considering the properties contrast between the two reservoir compartments, the model takes into account existence of transmissibility barriers with the use of resistance terms.
\end{abstract}

The model is used to analyze production performance of unconventional reservoirs, where the multistage fracturing of horizontal wells effectively creates a Stimulated Reservoir Volume (SRV) with an enhanced permeability surrounded by a non-stimulated region. It can also be used for analysis of compartmentalized conventional reservoirs. The analytical solutions provide type curves through which the controlling reservoirs parameters of a compartmentalized system can be estimated. The contribution of the supporting compartment is modeled based on a boundary dominated flow assumption. The transient behaviour of the support compartment is captured by application of "distance of investigation" concept. The model shows that depletion of the production and support compartments exhibit two unit slopes on a log-log plot of pressure versus time for CTR. For CTP, however, the depletions display two exponential declines. The depletion signatures are separated by transition periods, which depend on the contribution of the support compartment (i.e. transient or boundary dominated flow).

The developed equations can be implemented easily in a spreadsheet application, and are corroborated with the use of a numerical simulation. The study provides a new and nonconventional insight into the use of production data as an aid in the understanding of hydraulically fractured tight formations and compartmentalized conventional reservoirs.

Résumé - Nouvelle méthode d'analyse de la performance des réservoirs compartimentés Cet article présente un modèle analytique simple pour analyser la performance des réservoirs compartimentés produisant un Débit Final Constant (DFC) et une Pression Finale Constante (PFC). Ce modèle est basé sur un équilibre bien connu des matières et sur des équations d'écoulement soumis aux limites et est symbolisé par les critères de capacité et de résistance d'un compartiment de production et de réserve. Ces critères de capacité et de résistance constituent un ensemble de 
paramètres du réservoir qui permettent d'utiliser le modèle développé pour caractériser de tels systèmes. En plus de considérer les différentes propriétés des deux compartiments du réservoir, ce modèle prend en compte l'existence de barrières de transmissibilité en se servant des critères de résistance.

Ce modèle peut être utilisé pour analyser la performance de production de réservoirs non conventionnels où la fracturation multi-étape de puits horizontaux crée de manière efficace un Volume de Réservoir Stimulé (VRS) avec une perméabilité accrue, et entouré d'une zone non stimulée. Il peut également être utilisé pour l'analyse de réservoirs compartimentés conventionnels. Les solutions analytiques fournissent des courbes types, grâce auxquelles il est possible d'évaluer les paramètres de contrôle des réservoirs d'un système compartimenté. La contribution du compartiment de réserve est modélisée sur la base d'une hypothèse d'écoulement soumis aux limites. Le comportement transitoire du compartiment de réserve est pris en compte par l'application du concept de " distance d'étude ». Le modèle montre que l'épuisement des compartiments de production et de réserve est représenté sur un graphique logarithmique par deux courbes distinctes de pression et de temps pour le DFC. Pour la PFC cependant, les signes d'épuisement sont représentés par deux courbes de déclin exponentiel. Les signes d'épuisement sont séparés par des périodes de transition qui dépendent de la contribution du compartiment de réserve (c'est-à-dire écoulement transitoire ou écoulement soumis aux limites).

Les équations développées peuvent être facilement mises en œuvre dans un tableur, et sont corroborées grâce à l'utilisation d'une simulation numérique. Cette étude fournit une perspective nouvelle et non conventionnelle de l'utilisation des données de production en vue de mieux comprendre les réservoirs compacts traités par fracturation hydraulique et les réservoirs conventionnels compartimentés.

\section{NOMENCLATURE}

BDF Boundary Dominated Flow

c Compressibility, $1 / \mathrm{psi}$

C Fluid flow capacitance, Stb/psia

$C^{*} \quad$ Fluid flow capacity, Stb/(ft.psia)

CTP Constant Terminal Pressure

CTR Constant Terminal Rate

Der Bourdet derivative

$F_{c} \quad$ Ratio of capacitance of the production to support compartment

$F_{R} \quad$ Ratio of resistance of the production to support compartment

$F_{c}{ }^{*} \quad$ Ratio of capacity of the production to support compartment

$F_{R}{ }^{*} \quad$ Ratio of resistivity of the production to support compartment

$h \quad$ Net pay, $\mathrm{ft}$

$k \quad$ Permeability, md

$p_{\text {avg }}$

$p_{D}$

Average reservoir pressure within the compartment, psi

ing literature

$p_{D M}$

$p_{D M, B D F}$

$p_{D M}{ }^{c}$

$p_{D M}^{c s}$
Dimensionless pressure defined in this work

Dimensionless pressure at $\mathrm{BDF}$

Dimensionless pressure based on $\Delta p^{c}$

Dimensionless pressure based on $\Delta p^{c s}$ $p_{i} \quad$ Initial reservoir pressure, $\mathrm{psi}$

$p_{w f} \quad$ Wellbore flowing pressure, psia

$q \quad$ In situ well-production rate, bbl/D

$q_{D} \quad$ Dimensionless rate used in classical well testing literature

$q_{D d} \quad$ Dimensionless decline rate used in production data analysis literature

$q_{D M} \quad$ Dimensionless rate defined in this work

ODE Ordinary Differential Equation

$R \quad$ Flow resistance, $\mathrm{psi} / \mathrm{bbl}$

$s \quad$ (Subscription) support

SRV Stimulated Reservoir Volume

$t \quad$ Time, days

$t_{D} \quad$ Dimensionless time used in classical well testing literature

$t_{D M} \quad$ Dimensionless time

$t_{D M, B D F}$ Dimensionless time at BDF

$V \quad$ Volume, bbl

WFT Wireline Formation Tester

$x_{f} \quad$ Fracture half length, $\mathrm{ft}$

$y_{e} \quad$ Reservoir size in $y$-direction, $\mathrm{ft}$

$y_{e D} \quad$ Dimensionless distance

$y_{i n v} \quad$ Distance of investigation, $\mathrm{ft}$

$\Delta p^{c} \quad$ Pressure depletion of a dummy production compartment neglecting the support, psia

$\Delta p^{c s} \quad$ Pressure depletion of a dummy support compartment, psia 
$\alpha \quad$ Constant used for defining the distance of investigation

$\beta_{1} \quad$ Constant used for determining $q_{D}$ and $p_{D}$ (equal to $2 \pi \times 141.2$ in oil field units)

$\beta_{2} \quad$ Constant used for determining $t_{D}$ (equal to 0.00633 in oil field units)

$\lambda_{1} \quad$ First eigenvalue of the matrix of coefficients, A

$\lambda_{2} \quad$ First eigenvalue of the matrix of coefficients, A

$\mu \quad$ Viscosity, cp

$\phi \quad$ Porosity, fraction

\section{SI CONVERSION FACTORS}

$\mathrm{bbl} \times 1.589873 \mathrm{E}-1=\mathrm{m}^{3}$

cp $\times 1 \mathrm{E}-3=$ Pa.s

$\mathrm{ft} \times 3.048 \mathrm{E}-1=\mathrm{m}$

$\mathrm{mD} \times 9.869223 \mathrm{E} 16=\mathrm{m}^{2}$

psi $\times 6.894757 \mathrm{E} 3=\mathrm{Pa}$

* Conversion factors are exact

\section{INTRODUCTION}

Compartmentalized reservoirs are the result of segregation of a petroleum accumulation into a number of individual fluid/pressure compartments. They occur when flow is restricted across some form of boundaries in the reservoir. These boundaries are caused by a variety of geological features and processes and can be either completely sealed, across which no flow can occur, or partially sealed in which case the boundaries exhibit low to very low permeability (permeability barriers). The latter allows fluids and pressures of the compartments to equilibrate over geologic time-scales, and retards cross-flow between compartments during production. As a result, there will be pressure discontinuities across the reservoir, which according to Stewart and Whaballa (1989) can occur over near horizontal events such as shales or micaceous streaks and/or near vertical events such as partially communicating faults or turbidite lobe interfaces.

Evidence of compartmentalization has been observed in both oil and gas reservoirs (Junkin et al., 1992). A number of compartmentalized reservoirs have been discovered in different parts of the world including those in the North Sea (Fox et al., 1988), Texas Gulf Coast (Junkin et al., 1992) and Australia (Malavazos and McDonough, 1991). Stewart (2011) presents a well-test case study in a MiddleEast carbonate reservoir, where the derivative unit slope buildup indicates a charging-recharge mechanism. The compartmentalized behaviour can also occur in particular meandering fluvial reservoirs. In these environments, the depletion and the lateral cross-flow between the laterally avulsing point bar deposits separated by the semi-permeable channel fills can effectively create a compartmentalized system (Hamdi, 2014; Hamdi et al., 2014). Corbett et al. (2012) present an interesting well-test response in a meandering channelized system, where numerical well-test simulations and a multi-point statistics approach support the analytical well-test interpretation and the existence of effective sand bar compartments. Malavazos and McDonough (1991) demonstrate another channelized reservoir example where there is communication between the stacked channel compartments.

An important aspect of reservoir compartmentalization is a stacked channel realization where two sand bodies communicate hydraulically with each other through an overlapping area (interface). Chilingarian et al. (1992) give an account of geologic and engineering aspects of compartmentalization in carbonate reservoirs and point out that reservoir compartmentalization may result not only from vertical and lateral lithofacies changes, but also from spatial variations stemming from processes of post-depositional diagenesis that create secondary porosity in the rocks.

Reservoir compartmentalization can also be considered in unconventional reservoirs, where multi-frac completion of horizontal wells create an SRV region of enhanced permeability around the horizontal well that is surrounded by an unstimulated region of much lower permeability (Stalgorova and Mattar, 2012). It is therefore reasonable to model the production behaviour of such a reservoir using a model where the production and support tanks are attributed to the SRV and unstimulated reservoir sections, respectively.

\section{COMPARTMENT DETECTION AND ANALYSIS}

Reservoir compartmentalization is detected primarily from observations of pressure discontinuities both areally and vertically (Stewart and Whaballa, 1989). Such reservoir pressure variations over a producing field can be monitored using Wireline Formation Testers (WFT) and are due to differential depletion of the system caused by permeability barriers or reservoir heterogeneities of one kind or another (Stewart, 2011). Bradley and Powley (1994) indicate other ways for detection of reservoir compartmentalization, including the changes in mineralogy and drilling rates and also using electrical resistivity, sonic velocity and density of shales. Reservoir simulation is another method which can be used to detect compartmentalization. Because of its complexity and associated degrees of freedom, though, this method is not justified in most cases before identifying and quantifying the major flow units and barrier resistances using other methods (Rahman and Ambastha, 2000; Stewart and Whaballa, 1989; Aguilera, 2013). 
There are also well-testing and production data analysis methods which can contribute to an improvement of the understanding of geology. Analytical models and related solutions can lead to a simplified description of the geological heterogeneities around the well and can provide good insights into the significance of various parameters affecting the transport phenomena (Massonnat et al., 1993). Stewart and Whaballa (1989) argue that an extended drawdown test should be performed to detect the limits of the producing compartment. They develop type curves to identify the extent of faulted boundaries between compartments. By matching the late time data, they estimate the resistance to flow of fluid at the boundary (which they call transmissibility). Their approach is based on the use of single-phase material balance equations, the concepts of boundary pressure time delay and desuperposition to predict pressures over different flow regimes. Along the same lines, Rahman and Ambastha (2000) develop analytical 1D, 2D and 3D solutions for constant-rate transient flow in compartmentalized systems.

Stewart (2011) presents a mathematical method for well-test analysis of a two-cell compartmentalized system. He presents the material balance equations in dimensionless terms and discusses the existence of two derivative unit slopes in logarithmic scales indicating depletion of the well and support compartments. He considers the effect of compartment volumes, neglecting the variations in other reservoir parameters, e.g. permeability, between the compartments. This work does not involve the mathematical complexities of Rahman and Ambastha (2000) and is appealing from a practical point of view.

The primary objective of this paper is to obtain information on the contrast of rock and fluid properties from analysis of compartmentalized or composite systems using the capacitance and resistance terms introduced by Shahamat et al. (2014). Two tanks (and therefore two capacitance and resistance terms) are considered in the model - production and support.
The support tank feeds the production tank; therefore, its contribution starts after investigating the production tank. The length component in the capacitance and resistance of the support tank is first assumed to be constant, equal to the size of the support tank in the $y$-direction. This assumption implies instantaneous depletion (i.e. BDF) of the support tank. Based on this assumption and by using the fluid-flow and material balance equations for both tanks, simple analytical solutions are derived for constant-rate and constant-pressure production of a compartmentalized system. Using the distance of investigation equation in the capacitance and resistance terms, it is shown that the solutions can be easily modified to account for transient flow within the support tank. The derived mathematical solutions enable the generation of production type curves which can be used to characterize a compartmentalized system and obtain meaningful information related to the contrast in rock and fluid properties between the compartments. To the authors' best knowledge, no analytical model is available in the literature that discusses the constant-pressure production of compartmentalized systems, and that can be used for long-term production forecasting. The mathematical simplification described in this paper has the same spirit as the work documented by many authors attempting to replace a complex reservoir model by a simpler equivalent "upscaled" system (Valko et al., 2000; Kaviani et al., 2008; Sayarpour et al., 2008; Yousef et al., 2009; Noetinger, 2015).

\section{MATHEMATICAL DEVELOPMENT}

The mathematical framework of this study is based on the capacitance-resistance approach for a single-phase fluid. Adapted from Shahamat and Aguilera (2010), the configuration of the different sections of a compartmentalized reservoir is depicted using a tank model (Fig. 1a). The production tank is directly connected to the wellbore through

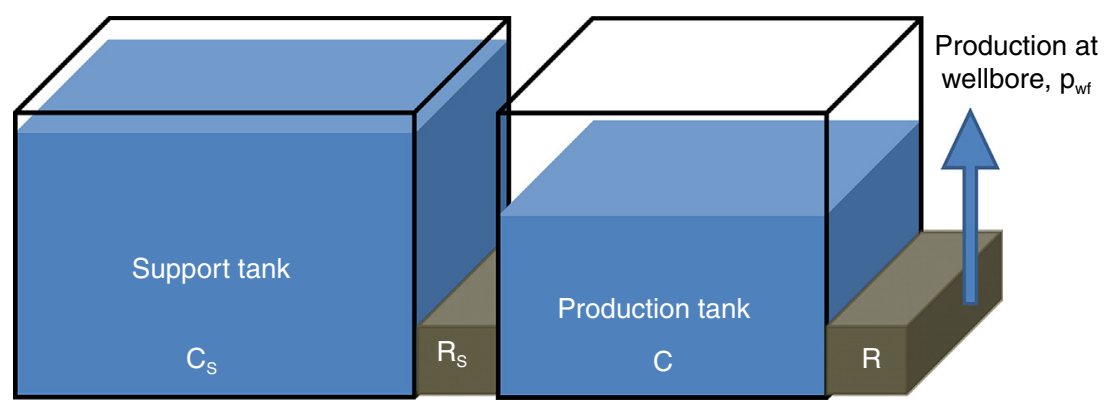

a)

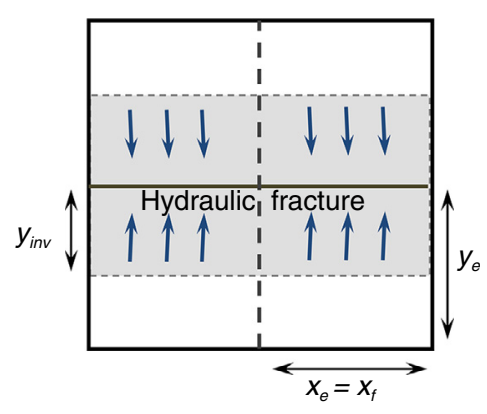

b)

Figure 1

a) Tank representation of a compartmentalized reservoir; b) a hydraulically fractured well in the center of a rectangular reservoir. 
the sand pack which implies a flow resistance $(R)$ to the wellbore. In addition, there is a support tank which is connected to the production tank through another barrier with a flow resistance, $R_{S}$. Depending on the values of flow capacitance $\left(C\right.$ and $\left.C_{S}\right)$ and resistance $\left(R\right.$ and $\left.R_{S}\right)$ there are various degrees of pressure communication between these compartments. Figure $1 \mathrm{~b}$ shows the base reservoir geometry of each resistance. This geometry implies pure transient linear flow and is chosen because linear flow has widespread application in unconventional reservoirs analysis.

It is assumed that the contribution of the support tank starts after investigating the production tank. This means that by the time the support tank starts feeding the production tank, the characteristics of the production tank (i.e. its $C, R$ and $t_{B D F}$ ) are known (Shahamat, 2014). These properties are used for modeling the compartmentalized reservoir behaviour.

Using the fluid-flow equation for relating the production rate to the average pressure of the production tank, one gets:

$$
q=\frac{1}{R}\left(p_{\text {avg }}-p_{w f}\right)
$$

Resistance, $R$, in the above equation is equivalent to the inverse of the productivity index. Knowing that the pressure at the adjoining sides of the production tank is average pressure $\left(p_{\text {avg }}\right)$, one can use the Darcy's equation for linear flow to analytically derive an equation for resistance according to $R=\left(\frac{\beta_{1} B \mu}{k h}\right)\left(\frac{y_{e}}{x_{f}}\right)$. In this equation, $\beta_{1}$ is a constant which is equal to $2 \pi \times 141.2$ in oilfield units and $B$ is the dimensionless formation volume factor.

For relating the average pressure of the support tank with the average pressure of the production tank we use Darcy's equation through the following formula:

$$
q_{s}=\frac{1}{R_{s}}\left(p_{\text {avg }, s}-p_{\text {avg }}\right)
$$

Linear flow regime within the support resistance leads to the following definition, $R_{s}=\left(\frac{\beta_{1} B \mu}{k h}\right)_{s} \times\left(\frac{y_{e}}{x_{f}}\right)_{s}$. In the above two equations, $q$ and $q_{s}$ are in situ rates.

In addition to Equations (1) and (2) for describing the behaviour of a compartmentalized system, the material balance equations for both tanks are employed. Material balance equation (i.e. compressibility equation) for the production tank is written in the following form:

$$
C \frac{d p_{a v g}}{d t}=q_{s}-q
$$

Similar to Equation (3), the compressibility equation for the support tank takes the following form:

$$
C_{s} \frac{d p_{\text {avg }, s}}{d t}=-q_{s}
$$

In the above two equations, capacitances $\left(C\right.$ and $\left.C_{S}\right)$ are expressed in Stb/psia. It is noted that capacitance is the multiplication of pore volume and total compressibility.

Equations (1) through (4) are the building blocks of the proposed methodology. Solutions to different production constraints (CTR or CTP) are obtained by defining different dimensionless parameters, as shown in the following sections.

\subsection{Constant Terminal Rate Production (Reservoir Limit Testing)}

For the CTR production, the dimensionless pressure and time, $p_{D M}$ and $t_{D M}$, are defined as follows (see Appendix C):

$$
p_{D M}=\frac{p_{i}-p}{q \times R}
$$

and,

$$
t_{D M}=\frac{t}{C \times R}
$$

It is noted that the dimensionless pressure defined in Equation (5) is different from the definition used in the classical well-testing literature. In well-testing literature, the dimensionless pressure is defined based on wellbore pressure, whereas Equation (5) is written in terms of average reservoir pressure (to be used in Eq. 1 through Eq. 4).

Moreover, two additional dimensionless parameters are introduced to characterize reservoirs with two communicating compartments. The first parameter, $F_{R}$, is the ratio of resistances, and reflects the combined effect of contrast in permeability, viscosity, formation thickness and skin factor, between the production and support tanks:

$$
F_{R}=\frac{R}{R_{S}}
$$

The second parameter, $F_{c}$, is the ratio of flow capacitances, and reflects the combined effect of changes in reservoir pore volume and compressibility of the production tank with respect to the support tank:

$$
F_{c}=\frac{C}{C_{s}}
$$

While normally one would expect to define the ratios of resistance and capacitance with respect to the production tank (i.e. $\frac{R_{s}}{R}$ and $\frac{C_{s}}{C}$ ), defining $F_{R}$ and $F_{c}$ according to Equations (7) and (8) simplify the formulations and obtained solutions in a concise form. As such, smaller values of $F_{R}$ (or $F_{c}$ ) indicate larger values of resistance (or capacitance) of the support tank compared with the production tank. 
Using the dimensionless variables defined in Equation (5) through (8), and upon some mathematical manipulations, Equations (3) and (4) take the following dimensionless forms, respectively:

$$
\begin{aligned}
& \frac{d p_{D M}}{d t_{D M}}=F_{R}\left(p_{D M s}-p_{D M}\right)-1 \\
& \frac{d p_{D M s}}{d t_{D M}}=-F_{R} F_{c}\left(p_{D M s}-p_{D M}\right)
\end{aligned}
$$

In these equations, $p_{D M}$ and $p_{D M s}$ are the dimensionless pressures of the production and support compartments. Equations (9) and (10) construct a system of linear differential equations, which can be solved to obtain the pressure of the support and production tanks. This system can be solved using matrix algebra routines. The detailed procedure for the solution is given in Appendix A. The resulting dimensionless pressure of the production tank $\left(p_{D M}\right)$ is given by the following closed form:

$$
\begin{aligned}
p_{D M}= & p_{D M, B D F}+\left(1+\frac{F_{R}}{\lambda_{2}}\right)\left(t_{D M}-t_{D M, B D F}\right) \\
& +\frac{F_{R}}{\lambda_{2}}\left(\frac{1}{\lambda_{2}}+p_{D M, B D F}\right)\left(1-e^{\lambda_{2}\left(t_{D M}-t_{D M, B D F}\right)}\right)
\end{aligned}
$$

and the solution for the support tank $\left(p_{D M s}\right)$ is:

$$
\begin{aligned}
p_{D M s}=\left(1+\frac{F_{R}}{\lambda_{2}}\right)\{ & \left(t_{D M}-t_{D M, B D F}\right)+\left(\frac{1}{\lambda_{2}}+p_{D M, B D F}\right) \\
& \left.\times\left(1-e^{\lambda_{2}\left(t_{D M}-t_{D M, B D F}\right)}\right)\right\}
\end{aligned}
$$

where, $\lambda_{2}=-F_{R}\left(1+F_{c}\right)$ is the smallest eigenvalue of the matrix of coefficients for the system of Equations (9) and (10). In these equations, $t_{D M, B D F}$ and $p_{D M, B D F}$ are the dimensionless time and pressure at the beginning of the BDF for the production tank.

\subsection{Constant Terminal Pressure Production (Rate Transient Analysis)}

For the CTP production, the dimensionless rate variable, $q_{D M}$, is defined as follows (see Appendix C):

$$
q_{D M}=\frac{q \times R}{p_{i}-p_{w f}}
$$

The dimensionless rate defined in this equation is based on the average reservoir pressure and therefore is the equivalent linear flow form of the Fetkovich rate decline parameter (Fetkovich, 1980). Employing the dimensionless rate (Eq. 13) and time (Eq. 6) in Equations (1) through (4), the constant-pressure behaviour of the compartmentalized system is obtained based on the solution of a system of ordinary differential equations:

$$
\begin{gathered}
\frac{d q_{D M}}{d t_{D M}}=-q_{D M}+q_{D M s} \\
\frac{d q_{D M s}}{d t_{D M}}=F_{R} q_{D M}-\left(F_{R}+F_{c} F_{R}\right) q_{D M s}
\end{gathered}
$$

In these equations, $q_{D M}$ and $q_{D M s}$ are dimensionless rates for the production and support compartments. Similar to the constant terminal rate solution, these equations can be solved using matrix algebra routines. The detailed procedure for the solution is outlined in Appendix B. The resulting dimensionless rate of the production tank $\left(q_{D M}\right)$ is given as following closed form:

$$
\begin{aligned}
q_{D M}=q_{D M, B D F}\{ & \left(\frac{\lambda_{2}+1}{\lambda_{2}-\lambda_{1}}\right) \times e^{\lambda_{1}\left(t_{D M}-t_{D M, B D F}\right)} \\
& \left.-\left(\frac{\lambda_{1}+1}{\lambda_{2}-\lambda_{1}}\right) \times e^{\lambda_{2}\left(t_{D M}-t_{D M, B D F}\right)}\right\}
\end{aligned}
$$

Similarly, the solution for the support tank $\left(q_{D M S}\right)$ is obtained according to Equation (17):

$$
\begin{aligned}
q_{D M S}= & q_{D M, B D F}\left(\frac{\left(\lambda_{1}+1\right)\left(\lambda_{2}+1\right)}{\left(\lambda_{2}-\lambda_{1}\right)}\right) \\
& \times\left\{e^{\lambda_{1}\left(t_{D M}-t_{D M, B D F}\right)}-e^{\lambda_{2}\left(t_{D M}-t_{D M, B D F}\right)}\right\}
\end{aligned}
$$

We note that in these two equations, $\lambda=\frac{-\left(1+F_{R}\left(1+F_{c}\right)\right) \pm \sqrt{\left(1-F_{R}\left(1+F_{c}\right)\right)^{2}+4 F_{R}}}{2}$, adopting the convention that $\lambda_{1}$ and $\lambda_{2}$ correspond to positive $(+)$ and negative $(-)$ signs, respectively. In these equations, $t_{D M, B D F}$ and $q_{D M, B D F}$ are the dimensionless time and rate at the beginning of the BDF for the production tank.

\section{RESULTS AND DISCUSSION}

In order to discuss the behaviour of the obtained equations in a systematic manner, first the equations obtained for the CTR production are elaborated and after that the CTP production behaviour is discussed.

\subsection{CTR Production}

The CTR behaviour of a compartmentalized reservoir can be better understood using the principle of superposition. The pressure change in the production tank is expressed 

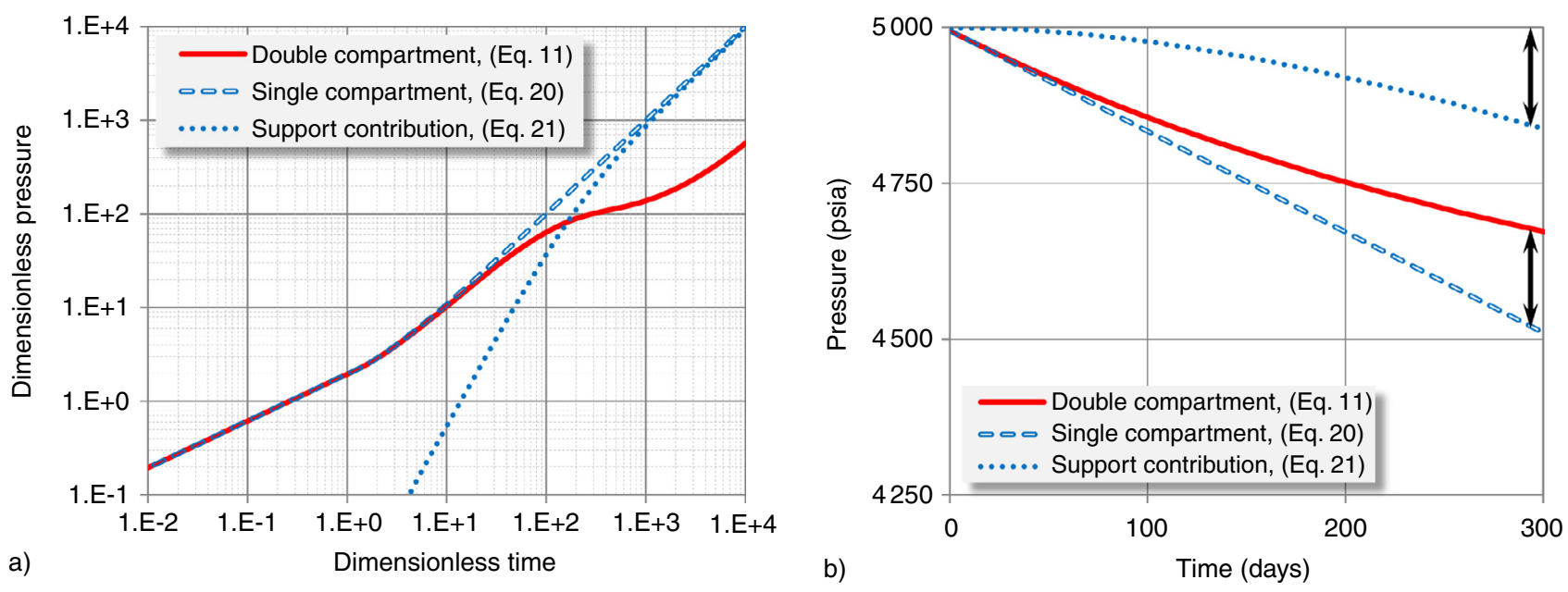

Figure 2

a) Log-log and b) Cartesian plots showing comparison of pressure behaviour of a single tank model versus a 2-compartment reservoir, $F_{R}=0.01$ and $F_{c}=0.1$. Also shown is the contribution of the support tank.

as the depletion of a single tank without any support $\left(\Delta p^{c}\right)$ subtracted by the pressure maintenance caused by the support tank $\left(\Delta p^{c s}\right)$. This means that the pressure change of a single tank can be expressed as below:

$$
\Delta p=\Delta p^{c}-\Delta p^{c s}
$$

Writing the above equation in dimensionless form, we obtain:

$$
p_{D M}{ }^{c s}=p_{D M}{ }^{c}-p_{D M}
$$

As evident from the definition of $p_{D M}{ }^{c}$, an equation for its behaviour can be obtained by using Equation (11), when $R_{S}$ approaches infinity and therefore $F_{R}=0$. This leads to the following relation:

$$
p_{D M}{ }^{c}=p_{D M, B D F}+\left(t_{D M}-t_{D M, B D F}\right)
$$

Using Equations (20) and (11) inside Equation (19), we get:

$$
\begin{gathered}
p_{D M}^{c s}=-\frac{F_{R}}{\lambda_{2}}\left(t_{D M}-t_{D M, B D F}\right)-\frac{F_{R}}{\lambda_{2}}\left(\frac{1}{\lambda_{2}}+p_{D M, B D F}\right) \\
\times\left(1-e^{\lambda_{2}\left(t_{D M}-t_{D M, B D F}\right)}\right)
\end{gathered}
$$

Note that this equation is different from Equation (12) and is useful for illustrating the contribution of the support tank. Figure 2 demonstrates the behaviour of a compartmentalized reservoir and the support contribution compared with a single tank reservoir model. The plot on the left hand side of this figure shows dimensionless pressure versus dimensionless time in logarithmic scales while the right hand side of the figure gives the Cartesian plot of pressure versus time. It is evident from these figures that during transient flow, the behaviour of both single and double tank models are identical. However, the effects of the support tank are felt after reaching the boundaries of the production tank. During this period, the log-log plot gives a straight line of unit slope for the single tank model which corresponds to a straight line on the Cartesian plot shown on the right hand side of Figure 2. Deviation from this behaviour (unit slope on the log-log plot or late time straight line on the Cartesian plot) is due to the pressure contribution of the support tank. The double-sided black arrows on the Cartesian plot clearly demonstrate that the amount of deviation is equal to the support contribution. It is noted that at late times, i.e. total system pseudo-steady state, the two compartments are depleted at the same rate with a constant pressure difference between them. Subtracting Equation (12) from Equation (11) and evaluating the result at late time gives the pressure difference according to the following relation:

$$
p_{D M}-p_{D M s}=-\frac{1}{\lambda_{2}}
$$

One can also determine the points where there are major changes in the plot of pressure versus time. These points include the start of BDF for the production tank, the time where deviation from the first unit slope on the log-log plot of $p_{D M}$ versus $t_{D M}$ takes place, and the time attributed to the start of the total system behaviour. The equation for the 
distance of investigation is used to identify the time at which these changes occur in the behaviour of a compartmentalized reservoir:

$$
y_{i n v}=\alpha_{1} \sqrt{\frac{\beta_{2} k t}{(\phi \mu c)_{t}}}
$$

In this equation, $k$ is the permeability in $\mathrm{md}, \mu$ is the viscosity in $\mathrm{cp}, \phi$ is the porosity, $c$ is the compressibility in $1 / \mathrm{psia}$ and time is in days. $\beta_{2}=0.00633$ and $\alpha_{1}$ is a constant which depends on the criterion used for defining the distance of investigation. $\alpha_{1}$ depends on the type of production at the wellbore. Wattenbarger et al. (1998) proposed $\alpha_{1}=1.42$ and 2 for constant-rate and constant-pressure production, respectively. Later, Nobakht and Clarkson (2012) suggested a modification of the distance of investigation equation over that of Wattenbarger et al. (1998) for constant-pressure production, giving $\alpha_{1}=2.55$. In this study and based on simulation studies, we use $\alpha_{1}=1.76$ and 2.23 for constant-rate and constant-pressure production, respectively. It should be mentioned that the definition of $y_{i n v}$ in the above equation is applicable only for $t<t_{B D F}$, where $t_{B D F}$ is the time to reach BDF. This upper limit of time for $y_{i n v}$ ensures that the calculated reservoir distance in the $y$-direction is limited to the size of the reservoir, $y_{e}$.

Using Equation (23), one can identify the time at which these changes occur in the behaviour of a compartmentalized reservoir. Writing this equation in terms of dimensionless variables defined in this work, one can obtain the time at which BDF of the production tank starts:

$$
t_{D M, B D F}=\frac{\pi}{2} \frac{1}{\alpha_{1}^{2} \alpha_{2}}
$$

The time where deviation from the single tank behaviour happens is determined from the following equation:

$$
t_{D M}=t_{D M, B D F}\left(\frac{F_{R}+1}{F_{R}}\right)
$$

and the time at which the total system BDF occurs can be determined using the distance of investigation equation for the total system:

$$
t_{D M}=t_{D M, B D F}\left(\frac{F_{R} F_{c}+1}{F_{R} F_{c}}\right)
$$

In addition to the above characteristic points, we can calculate the well-testing or semilog derivative (Der) of Equation (11) according to the following equation:

$$
\begin{aligned}
\operatorname{Der}=(1+ & \left.\frac{F_{R}}{\lambda_{2}}\right) t_{D M}-F_{R}\left(\frac{1}{\lambda_{2}}+p_{D M, B D F}\right) \\
& \times t_{D M} e^{\lambda_{2}\left(t_{D M}-t_{D M, B D F}\right)}
\end{aligned}
$$

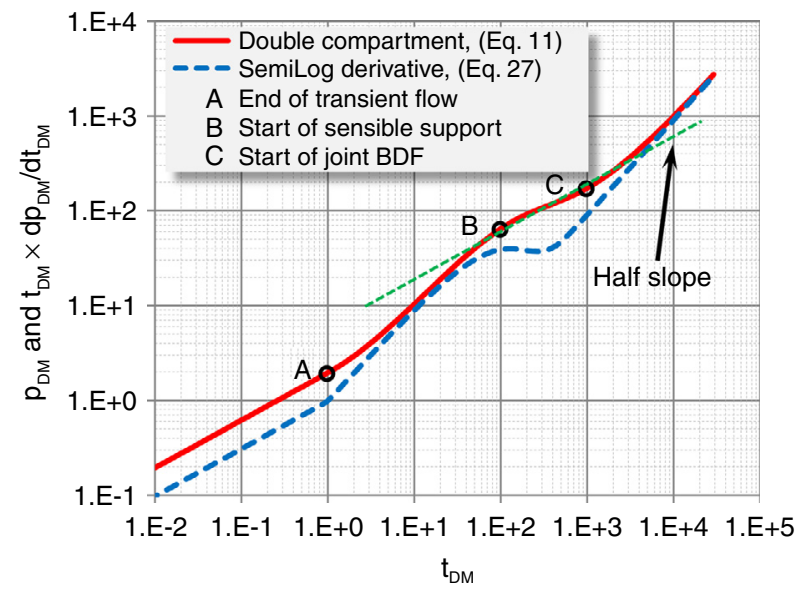

Figure 3

Log-log plot of $p_{D M}$ and well-testing derivative (Der) versus $t_{D M}$, along with the position of the characteristic points for the behaviour of the compartmented reservoir.

Figure 3 shows a plot of dimensionless pressure and the well-testing derivative versus dimensionless time with inclusion of the points at which the transient flow in the production tank ends (point A), the support tanks becomes active (B) and the BDF for the composite system begins (C). This figure demonstrates the powerful ability of the well-testing derivative for detecting different flow regimes. Compared with the dimensionless pressure plot, the well-testing derivative shows the start of joint BDF at a much earlier time. In addition, it is noted that while the dimensionless pressure curve manifests a half slope during the transition period between points $\mathrm{B}$ and $\mathrm{C}$, the welltesting derivative does not exhibit a half slope and therefore indicates that the flow regime during this period is not linear flow. This powerful diagnostic feature of derivative plot is used to advantage in well-test analysis for the detection of different flow regimes. In the context of production data analysis, however, the sensitive nature of the derivative amplifies the inherent noise in the production data so much that it makes the derivative plot essentially useless. Accordingly, it is reasonable to use the developed pressure solutions (rather than their derivatives) to make acceptable production analysis and therefore use the points with significant changes in the production behaviour to simply characterize a compartmentalized system (via Eqs. 24-26).

Evident from Equation (11), the behaviour of the double compartment model is a function of the value of $F_{c}$ and $F_{R}$. These parameters determine the time at which the straight line attributed to the behaviour of the total system takes place. Figure 4 shows the effect of $F_{c}$ and $F_{R}$ on the behaviour of the double tank model. As mentioned previously, 

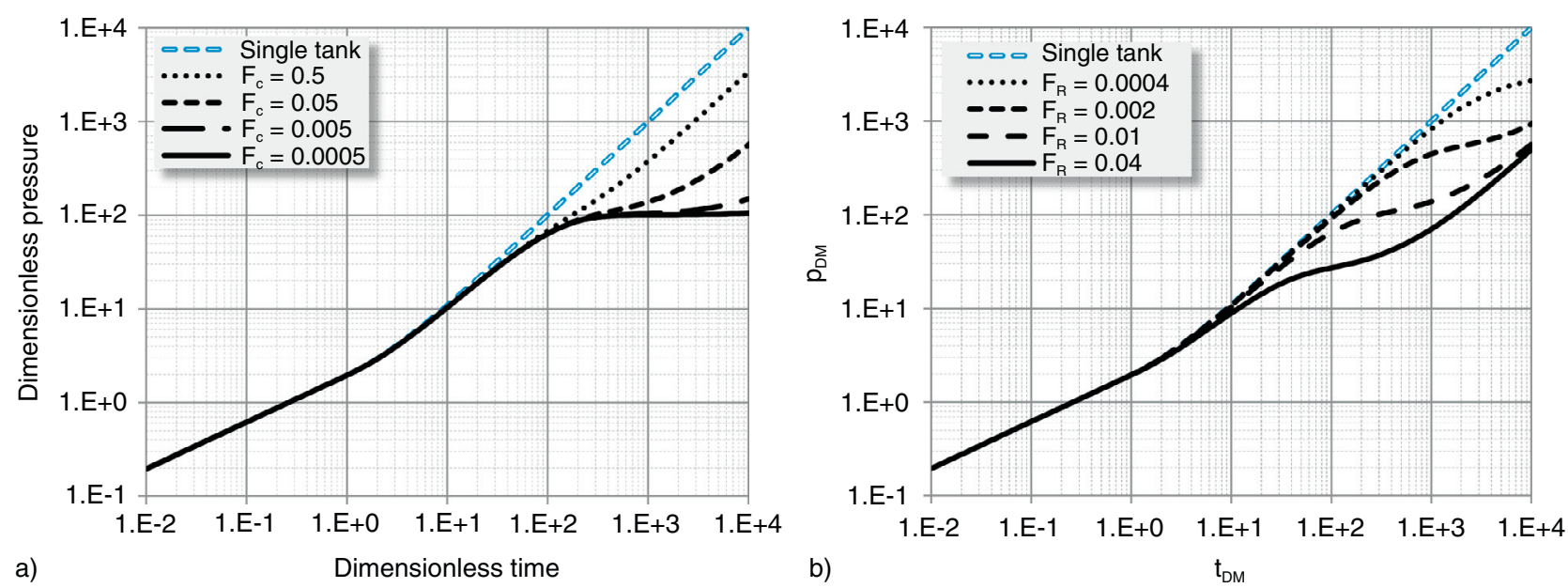

Figure 4

a) Effect of $F_{c}$ on the CTR response of a compartmentalized system with $F_{R}=0.01 ; \mathrm{b}$ ) effect of $F_{R}$ on the CTR response of a compartmentalized system with $F_{c}=0.05$.

smaller values of $F_{R}$ (or $F_{c}$ ) indicate larger resistance (or capacitance) of the support tank compared with the production tank and therefore later appearance of the total system unit slope. The plot on the left-hand side of this figure shows the effect of $F_{c}$ for a constant value of $F_{R}$ and vice versa for the plot on the right hand side of this figure.

Using Equation (11), type curves for different values of $F_{c}$ and/or $F_{R}$ can be easily generated which, through a simple type curve matching approach, can be used for estimating the contrast of capacitance $\left(F_{c}\right)$ and resistance $\left(F_{R}\right)$ between production and support compartments. These parameters can be used to compare the hydraulic diffusivity of the two reservoir sections.

\subsection{CTP Production}

CTP production from a two-compartment reservoir yields a double exponential decline in rate; each is attributed to depletion of one compartment. A log-log plot of dimensionless rate versus dimensionless time (Eq. 16) for typical values of $F_{R}=0.01$ and $F_{c}=0.05$ is given in Figure 5. This figure demonstrates the difference between the behaviour of a single tank and that of a two-tank reservoir. Depletion of a single tank results in the well-known exponential decline (Fetkovich, 1980) which can be obtained by substitution of $\lambda_{1}=0$ and $\lambda_{2}=-1$ in Equation (16):

$$
q_{D M}=q_{D M, B D F} \times e^{-\left(t_{D M}-t_{D M, B D F}\right)}
$$

Based on these parameters and using the equation for the distance of investigation, the points where major changes

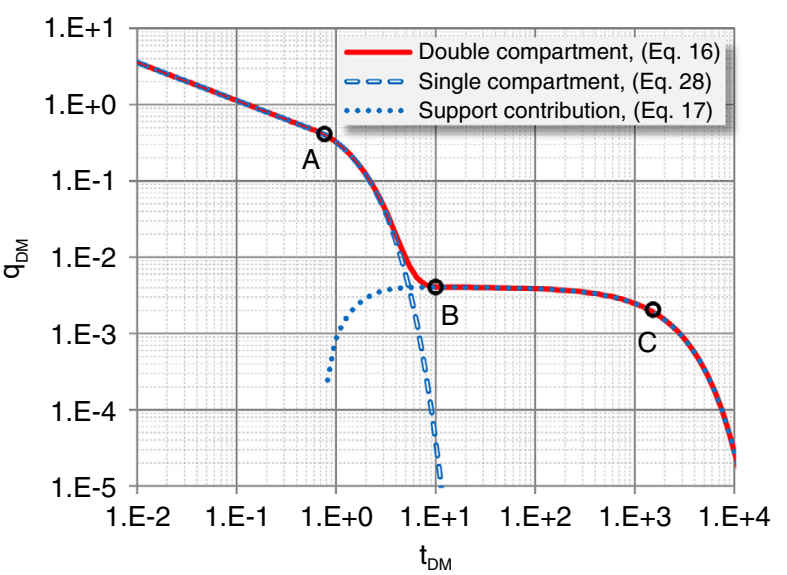

Figure 5

Log-log plot of $q_{D M}$ versus $t_{D M}$ showing the behaviour of a double tank model, $F_{R}=0.01$ and $F_{c}=0.05$, the position of its characteristic points and its comparison with the single tank model.

take place in the plot of rate versus time can be determined. The first point is the start of the BDF for the production tank, which as in the case of CTR can be determined with the use of Equation (24). The second point is the time where deviation from the single tank behaviour happens. This can be determined according to the equation (Shahamat, 2014):

$$
t_{D M}=t_{D M, B D F}+\frac{1}{\lambda_{1}+1} \times \ln \left(\frac{\lambda_{2}-\lambda_{1}}{\lambda_{2}+1}\right)
$$



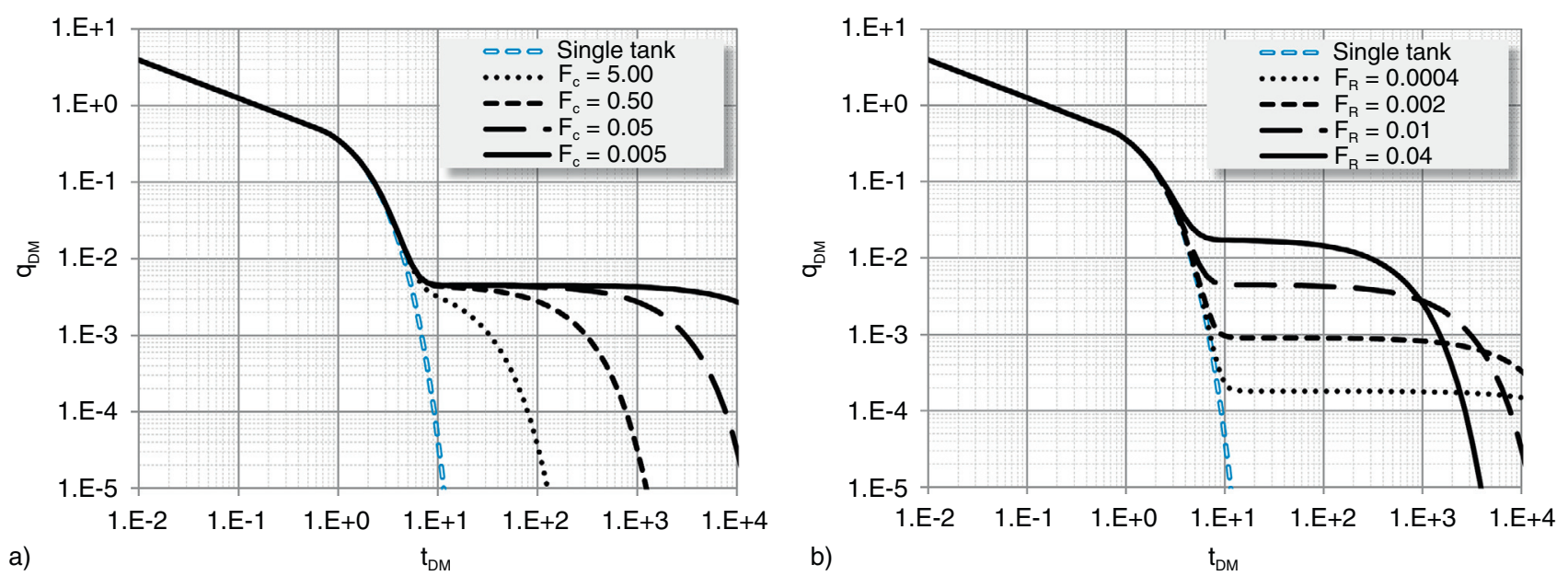

Figure 6

a) Effect of $F_{c}$ on the CTP response of a compartmentalized system with $F_{R}=0.01 ; \mathrm{b}$ ) effect of $F_{R}$ on the CTP response of a compartmentalized system with $F_{c}=0.05$.

The time for the total system BDF can be determined using the distance of investigation equation for the total system, similar to the case of CTR production, Equation (26).

Figure 5 shows the location of the characteristic points (end of transient flow in the production tank (A), start of sensible support tank contribution (B) and start of BDF for the composite system (C)) on the log-log plot of $q_{D M}$ versus $t_{D M}$ for $F_{R}=0.01$ and $F_{c}=0.1$. It depicts that the rate decline associated with such a compartmentalized system exhibits a period following depletion of the production tank where the flow rate is constant. $q_{D M}$ for this period can be obtained from the second exponential term in Equation (16).

Similar to the CTR, deviation from the single tank behaviour is because of the pressure contribution of the support tank and is a function of the values of $F_{c}$ and $F_{R}$. These parameters determine the behaviour of the system. Figure 6 shows the effect of $F_{c}$ and $F_{R}$ on the behaviour of the double tank model. The plot on the left-hand side of this figure shows the effect of $F_{c}$ for a constant value of $F_{R}$ and vice versa for the plot on the right of this figure.

Using Equation (16), type curves for different values of $F_{c}$ and/or $F_{R}$ can be easily generated which can be used through a simple type curve matching approach to estimating the contrast of capacitance $\left(F_{c}\right)$ and resistance $\left(F_{R}\right)$ between production and support compartments. These parameters reflect the effective variation of reservoir and fluid properties across the compartments.

\section{TRANSIENT FLOW WITHIN THE SUPPORT TANK}

The equations developed so far are based on the premise of instantaneous (or relatively instantaneous) depletion of the support tank. This means that in the above equations the transient flow in the support tank is ignored. As a result, immediately after the start of support tank contribution, its flow regime is assumed to be BDF. This is a reasonable assumption for cases where the size of the support and production tanks and also their permeabilities are very close to each other. However in cases where the size of the support tank is bigger than that of the production compartment, the support permeability should be higher in order to satisfy its relative instant depletion. In practice, this may not be the case and the production tank may be surrounded by a number of lower (instead of higher) permeability sections. Moreover, having a lower-permeability support tank would require extended transient flow to be considered.

In order to extend the application of the proposed model for capturing transient flow within the support tank, the distance of investigation concept is employed. The length components in the capacitance and resistance parameters of the support tank are allowed to change with time according to the distance of investigation equation, Equation (23), until reaching its BDF. This implies that parameters $F_{c}$ and $F_{R}$ decrease with time and become constant only at late times, therefore they cannot be used for type curve generation. For the purpose of producing the appropriate type curves, we define $F_{c}{ }^{*}$ and $F_{R}{ }^{*}$ where $F_{c}{ }^{*}=F_{c} \times \frac{y_{i v v s}}{y_{e}}$ and $F_{R}{ }^{*}=F_{R} \times \frac{y_{i v y_{s} s}}{y_{e}}$. These parameters indicate the ratios of 

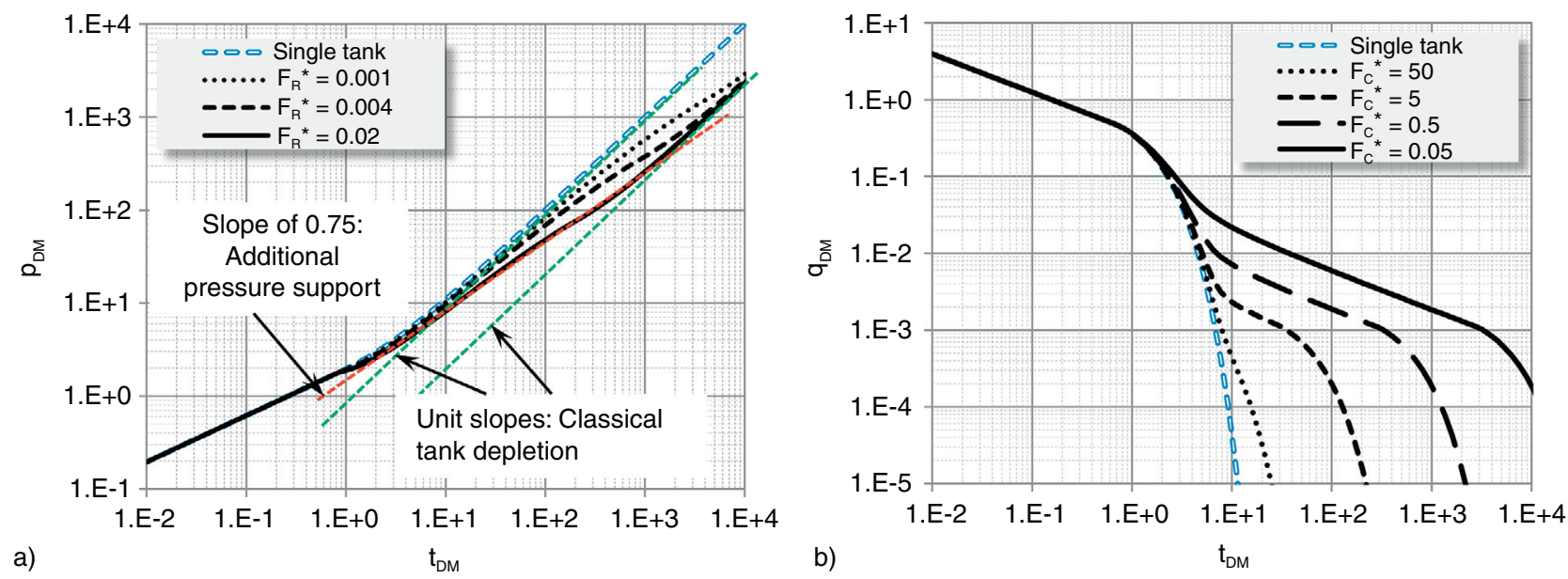

Figure 7

a) Effect of $F_{R}{ }^{*}$ on the CTR response of a compartmentalized system with $F_{c}{ }^{*}=0.1$; b) effect of $F_{c}{ }^{*}$ on the CTP response of a compartmentalized system with $F_{R}{ }^{*}=0.005$.

the capacity and resistivity of the production to support tank, rather than their capacitance and resistance, and exclude the effect of variable distance introduced by $y_{i n v}$ in the support compartment (Shahamat et al., 2014). Using these parameters and just for demonstration purposes, the obtained CTR and CTP type curves for typical values of $F_{R}{ }^{*}$ and $F_{c}{ }^{*}$ are shown in Figure 7.

Figure 7a is the CTR response of a compartmentalized system considering the effect of transient flow within the support tank. It shows that contribution of the support tank can considerably alter the production signature in such a way that the classic half or unit slopes may not exist for long times. In this figure, there is one case with a slope of 0.75 during the transition period for almost $3 \log$ cycles. Figure $7 \mathrm{~b}$ depicts the effect of $F_{c}{ }^{*}$ on the CTP response of a reservoir. It illustrates two straight line of slope -0.5 on the log-log plot of $q_{D M}$ versus $t_{D M}$ indicating linear flow regime. Each of these linear periods is followed by significant drops in dimensionless rate that correspond to exponential declines signifying depletion in the associated compartment.

The proposed tank model and developed type curves presented in this work can be used to characterizing a compartmentalized or composite reservoir. In the following section, two different numerical simulation models with known compartment parameters are presented. Using the developed analytical models in this paper, we then estimate the compartments properties and compare them with the numerical models input data. Consistent results between the numerical simulation and the proposed analytical
TABLE 1

Reservoir and fluid properties used for constructing Case 1

\begin{tabular}{c|c|c}
\hline Parameter & Region 1 & Region 2 \\
\hline$k(\mathrm{md})$ & 0.5 & 0.01 \\
\hline$\Phi$ (fraction) & 0.04 & 0.06 \\
\hline$c_{f}\left(\mathrm{psi}^{-1}\right)$ & $3 \times 10^{-6}$ & $9 \times 10^{-6}$ \\
\hline
\end{tabular}

approach shows the validity and application of our developed solutions.

\section{SIMULATION EXAMPLE, CASE 1}

Using a commercial numerical simulator, Case 1 is a singlephase liquid composite reservoir with contrasting reservoir properties shown in Table 1 and reservoir schematic depicted in Figure 8. The production well is in the center of the higher permeability reservoir section, is hydraulically fractured and is put on production for about 12 years.

Constant-rate and constant-pressure production of the well results in pressure and rate data whose logarithmic plots are shown in Figure 9. Using the developed type curves in Figure 7 and through a matching process, the parameters are determined to be $F_{c}{ }^{*}=0.28$ and $F_{R}{ }^{*}=0.02$. Calculation of these parameters based on the simulation input data give identical values. This consistency validates the approach and developed equations for production data analysis of a composite reservoir. 


\section{SIMULATION EXAMPLE, CASE 2}

In this case, a compartmentalized reservoir with similar properties and reservoir configuration as the previous example is used. Compartmentalization is imitated using a thin lower permeability region between the two reservoir sections. Such a lower permeability region (which in this case is $5 \%$ of the lower permeability region) represents a fault or a low-permeability shale barrier and is used to simulate a compartmentalized reservoir. The production well in the

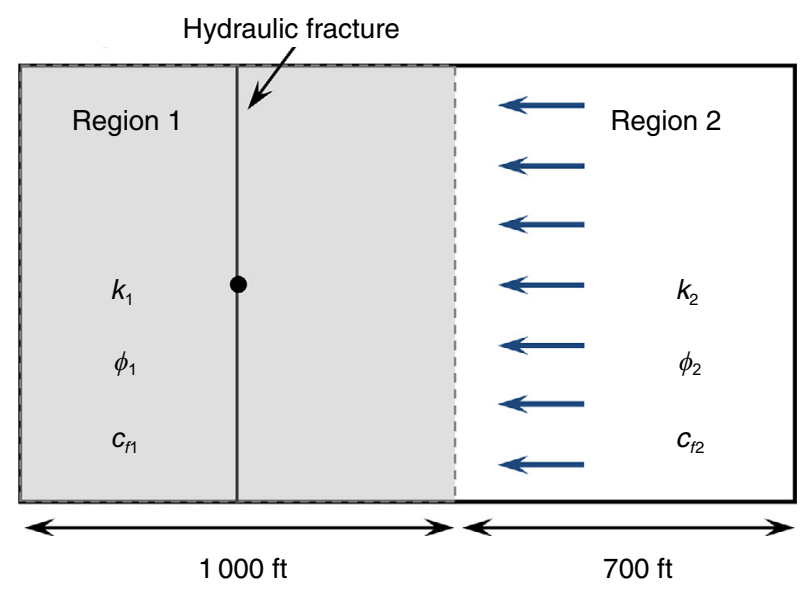

Figure 8

Reservoir geometry used for simulation of a composite reservoir.

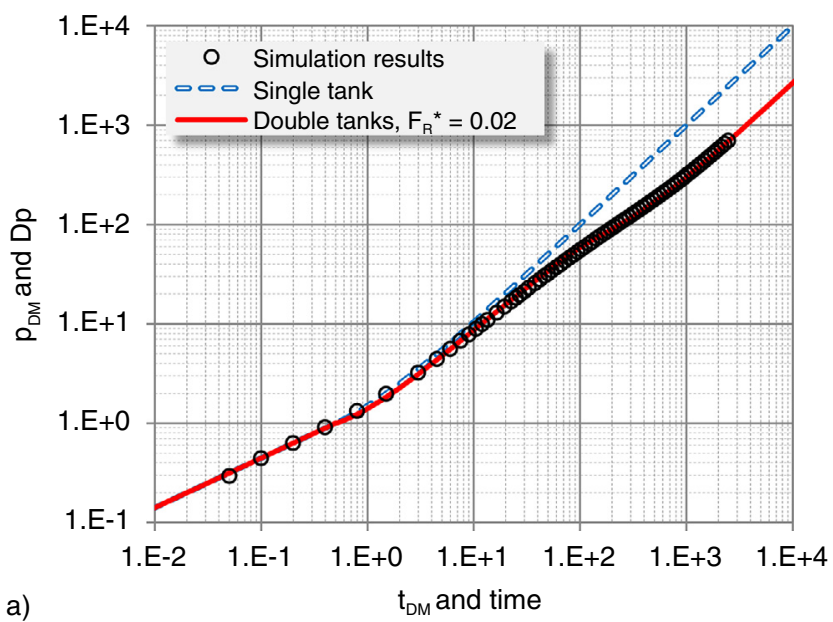

center of the higher permeability region is hydraulically fractured and is put on production for 12 years under both constant-rate and constant-pressure production.

Existence of the low-permeability barrier delays the production from the lower permeability region in such a way that its transient flow (half slope) is absent from the well's production signature. As a result, one can assume instantaneous support and simply use Equations (11) and (16) for its production analysis. For the CTR production data and using the type curves in Figure 4, we obtain a perfect match as illustrated in Figure 10 showing $F_{R}=1.2 \times 10^{-3}$ and $F_{c}=0.2$. It is noted here that $F_{R}$ represents the ratio of the flow resistance between the production and support compartments. For the simulation case defined in this example, it fuses the effect of a flow barrier (low-permeability region) in addition to the contrast of permeability between the two reservoir sections. This is an advantage of this method as it combines the effect of different factors affecting production at the wellbore in a single term to be used in the type curve analysis. On the other hand, $F_{c}$ represents the ratio of capacitance of production and support compartments. This term represents the combined effects of the drainage volume and compressibility associated with each compartment.

Analyzing the CTP production data and using Figure 6, the type curve matching (Fig. 10b) yields $F_{R}=2.3 \times 10^{-3}$ and $F_{c}=0.2$. Comparison of the matching parameters for CTP and CTR indicates different values of $F_{R}$ for the same compartmentalized system. The difference is attributed to the effect of transient flow within the support compartment for each of these production scenarios. In other words, as indicated in Equation (23), the propagation of pressure disturbance in the reservoir as a result of

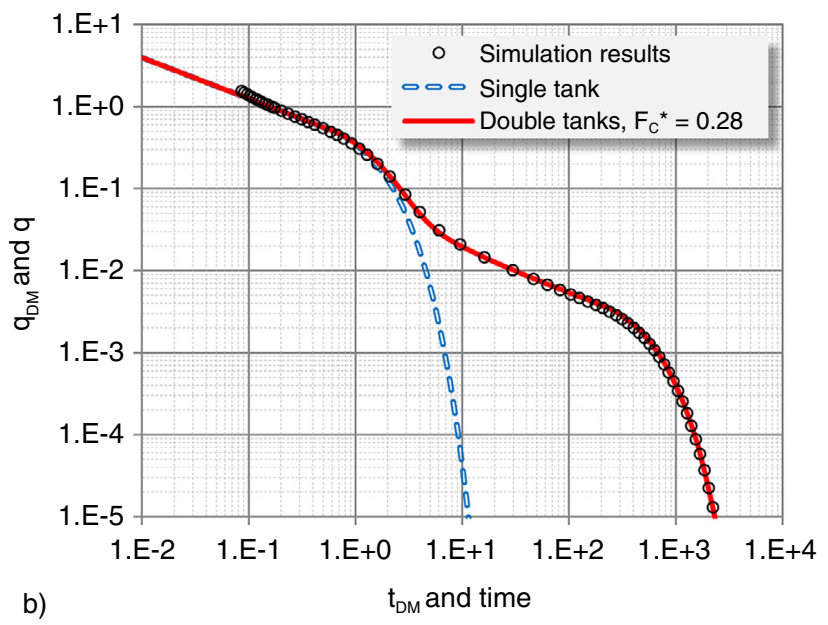

Figure 9

Type curve match results for Case 1; a) constant rate, b) constant pressure. 

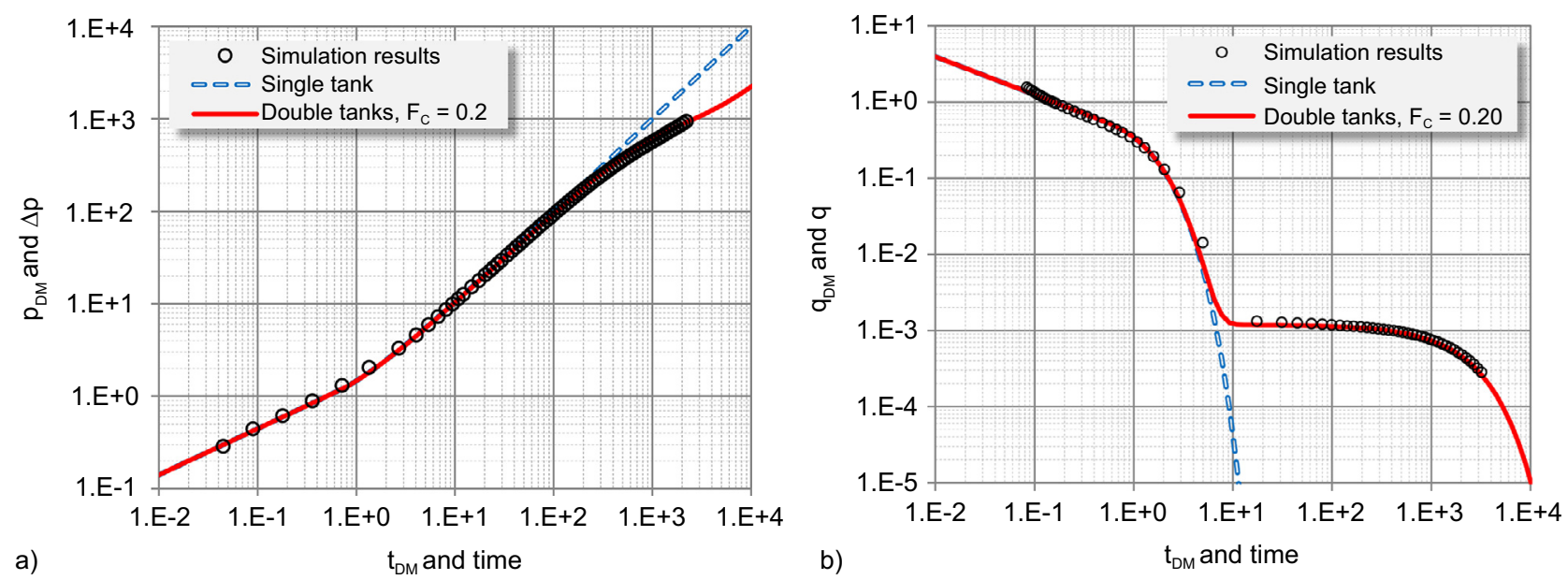

Figure 10

Type curve match results for Case 2; a) constant rate, b) constant pressure.

constant terminal rate and constant terminal pressure is different for systems that exhibit linear flow. This leads to different values of $F_{R}$ obtained from analysis of constant-rate and constant-pressure solutions.

\section{DISCUSSION}

Comparison of the results for the composite and compartmentalized reservoir models in the above examples shows that CTR and CTP of a composite model yield similar values for $F_{c}{ }^{*}$ and $F_{R}{ }^{*}$. However, for the compartmentalized model with BDF within the support tank, type curve matching of the CTR and CTP gives different values of $F_{R}$ for the same reservoir. This reveals that $F_{R}$ is not only dependent on the reservoir and fluid characteristics of the two compartments, but also on the production mechanisms. Intuitively, it has resemblance to the skin factor that is used extensively in well-testing analysis. The skin factor explains the additional pressure drop at the wellbore and has different effects for CTR compared with CTP. While the skin factor is defined as the ratio of the permeabilities and the sizes of damaged and undamaged zones, $F_{R}$ is the ratio of the permeability, viscosity and size of the production and support compartments.

Our extensive numerical experiments with different contrast in properties and with low-permeability barrier showed that not only the permeability and size of the support tank, but also its compressibility and porosity greatly influence the performance behaviour of a compartmentalized reservoir. For smaller values of hydraulic diffusivity of the support compartment, lower permeability barrier results in a production signature which can be adequately modeled using the simplified solution (with constant $F_{c}$ and $F_{R}$ ). In general, for a system with relatively equally-sized production and support regions and with a barrier that has smaller than $1 \%$ permeability of the lower permeability region, the BDF assumption of the support compartment is valid and its performance can be reasonably modeled using the developed solution with constant $F_{c}$ and $F_{R}$.

This study illustrates that the parameters $F_{c}$ and $F_{R}$ (or $F_{c}{ }^{*}$ and $F_{R}{ }^{*}$ ) can be used as parameters to characterize a compartmentalized/composite reservoir. Type curves can be generated for different values of $F_{c}$ and $F_{R}$ (or $F_{c}{ }^{*}$ and $F_{R}{ }^{*}$ ). Here it should be stressed that when analyzing production data, these parameters are not known a priori. Therefore type curve matching process may not be very practical. Although a type curve matching process was used for obtaining these parameters, a history matching process can be employed instead to determine $F_{c}$ and $F_{R}$ (or $F_{c}{ }^{*}$ and $\left.F_{R}{ }^{*}\right)$.

It is worth mentioning that even though $F_{c}$ and $F_{R}$ are based on physical properties (e.g. permeability, porosity, compressibility etc.) of the system, the history matched $F_{c}$ and $F_{R}$ may not reflect these exact properties but are more likely a combination of the various complexities in the system (transient flow, existence of barrier, constant-rate versus constant-pressure production, etc.). Nevertheless, because they are based on system properties, they are meaningful, and their value obtained from the history matching should be much more useful than a purely empirically derived match (as in the Arps curves). In essence, it can be stated that these parameters are matching parameters that qualitatively have physical meaning as they are related to physical 
properties of the system, but quantitatively can have different values under different production scenarios (as in the case of $F_{R}$ ).

The focus of this paper was on modeling of capacitance and resistance for the single-phase fluid systems. However, the approach can be extended for multiphase flow situations by employing proper pseudo variable (Behmanesh et al., 2015) definitions to linearize the equations. Therefore the single-phase liquid analogy can still be applied. This is the subject of another paper and is not discussed in this work.

\section{CONCLUSIONS}

In this work, the long-term performance of compartmentalized reservoirs under constant terminal rate and pressure production has been studied. Using the capacitance and resistance concepts, a mathematical model has been developed for compartmentalized reservoirs and detailed analytical solutions have been presented. Different scenarios for $\mathrm{BDF}$ and transient flow within the support cell are considered. The model allows for existence of leaky boundaries as well as different reservoir properties across the compartments. Key conclusions are as follows:

- the analytical solutions are obtained in real time domain and therefore can be easily implemented in a spreadsheet application through automatic fitting procedures and inversion loops for parameter estimation;

- depletion of each compartment is revealed by two unit slopes on a log-log plot of pressure versus time for CTR. For CTP, however, the depletions display two exponential declines. These depletion signatures are separated by transition periods which depend on the support cell contribution (i.e. BDF or transient);

- expressed in dimensionless forms, the solutions provide type curves which can be used through a matching process for estimating the contrast of capacitance and resistance (i.e. $F_{c}$ and $F_{R}$ or $F_{c}{ }^{*}$ and $F_{R}{ }^{*}$ ). These parameters reflect the combined effect of variation of reservoir and fluid properties across the compartments and control the flow performance of compartmentalized systems;

- the basic equations are developed based on the BDF as the prevailing conditions. However, the distance of investigation concept can be used to model the transient effect of the supporting compartment. This is considered as a generalization of two-region linear composite model with inclusion of a leaky interface (permeability barrier);

- the difference in distance of investigation for the CTR and CTP leads to different values of $F_{R}$ (for instantaneous depletion of the support) which are obtained from the type curve matching process of pressure and rates for the same system.

\section{REFERENCES}

Aguilera R. (2013) Flow Units: From Conventional to Tight Gas to Shale Gas to Tight Oil to Shale Oil Reservoirs, SPE Paper 165360 presented at the SPE Western Regional \& AAPG Pacific Section Meeting, Joint Technical Conference, Monterey, CA, USA, 19-25 April.

Behmanesh H., Hamdi H., Clarkson C.R. (2015) Production data analysis of tight gas condensate reservoirs, Journal of Natural Gas Science and Engineering 22, 0, 22-34.

Bradley J.S., Powley D.E. (1994) Pressure Compartments in Sedimentary Basins: A Review, in Basin Compartments and Seals, Ortoleva P.J. (ed.), The American Association of Petroleum Geologists.

Chilingarian G.V., Mazzullo S.J., Rieke H.H. (1992) Introduction, in Carbonate Reservoir Characterization: A Geologic-Engineering Analysis, Part I, Elsevier Science Publishers B.V.

Corbett P.W.M., Hamdi H., Gurav H. (2012) Layered fluvial reservoirs with internal fluid cross flow: a well-connected family of well test pressure transient responses, Petroleum Geoscience 18, 219-229.

Fetkovich M.J. (1980) Decline Curve Analysis Using Type Curves, Journal of Petroleum Technology, 32, 6, 1065-1077, SPE-4629-PA.

Fox M.J., Chedburn A.C.S., Stewart G. (1988) Simple Characterization of Communication Between Reservoir Regions, SPE Paper 18360 presented at the European Petroleum Conference, London, United Kingdom, 16-19 Oct.

Hamdi H. (2014) Well-test response in stochastic permeable media, Journal of Petroleum Science and Engineering 119, 169-184.

Hamdi H., Ruelland P., Bergey P., Corbett P.W.M. (2014) Using geological well testing for improving the selection of appropriate reservoir models, Petroleum Geoscience 20, 4, 353-368.

Junkin J.E., Sippel M.A., Collins R.E., Lord M.E. (1992) Well Performance Evidence for Compartmented Geometry of Oil and Gas Reservoirs, SPE Paper 24356 presented at the SPE Rocky Mountain Regional Meeting, Casper, Wyoming, USA, 18-21 May.

Kaviani D., Jensen J.L., Lake L.W., Fahes M. (2008) Estimation of Interwell Connectivity in the Case of Fluctuating Bottomhole Pressures, SPE Paper 117856 presented at the International Petroleum Exhibition and Conference, Abu Dhabi, 3-6 Nov, DOI: 10.2118/ 117856-MS.

Malavazos M., McDonough R.C.M. (1991) Pressure-Transient Response in Compartmentalised Gas Reservoirs: A South Australian Field Example, SPE Paper 23009 presented at the SPE Asia-Pacific Conference, Perth, Australia, 4-7 Nov.

Massonnat G.J., Norris R.J., Chalmette J.-C. (1993) Well Test Interpretation in Geologically Complex Channelized Reservoirs, SPE Paper 00026464 presented at the SPE Annual Technical Conference and Exhibition, Houston, Texas, 3-6 Oct.

Nobakht M., Clarkson C.R. (2012) A New Analytical Method for Analyzing Linear Flow in Tight/Shale Gas Reservoirs: ConstantFlowing-Pressure Boundary Condition, SPE Reservoir Evaluation \& Engineering 15, 3, 370-384, SPE-143989-PA.

Noetinger B. (2015) About the Determination of Quasi Steady State Storativity and Connectivity Matrix of Wells in 3D Heterogeneous Formations, Mathematical Geosciences (submitted).

Rahman N.M.A., Ambastha A.K. (2000) Generalized 3D Analytical Model for Transient Flow in Compartmentalized Reservoirs, SPE Journal 5, 3, 276-286, SPE-65106-PA. 
Sayarpour M., Kabir C.S., Lake L.W. (2008) Field Applications of Capacitance-Resistive Models in Waterfloods, SPE Paper 114983 presented at the SPE Annual Technical Conference and Exhibition, Denver, 21-24 Nov, DOI: 10.2118/114983-MS.

Shahamat M.S. (2014) Production Data Analysis of Tight and Shale Reservoirs, PhD Thesis, University of Calgary, Calgary.

Shahamat M.S., Aguilera R. (2010) A New Method for Production Decline Analysis of Tight Gas Formations, SPE Paper 138149 presented at the Canadian Unconventional Resources and International Petroleum Conference, Calgary, Alberta, Canada, 19-21 Oct.

Shahamat M.S., Mattar L., Aguilera R. (2014) A Physics-Based Method for Production Data Analysis of Tight and Shale Petroleum Reservoirs Using Succession of Pseudo-Steady States, SPE Paper 167686 presented at the SPE/EAGE European Unconventional Resources Conference and Exhibition, Vienna, Austria, 25-27 Feb.

Stalgorova E, Mattar L. (2012) Practical Analytical Model To Simulate Production of Horizontal Wells With Branch Fractures, SPE Paper 162515 presented at the SPE Canadian Unconventional Resources Conference, Calgary, Alberta, Canada, 30 Oct.-1 Nov.

Stewart G. (2011) Well Test Design \& Analysis, PennWell.
Stewart G., Whaballa A.E. (1989) Pressure Behavior of Compartmentalized Reservoirs, SPE Paper 19779 presented at the SPE Annual Technical Conference and Exhibition, San Antonio, Texas, USA, 8-11 Oct.

Valko P., Doublet L.E., Blasingame T.A. (2000) Development and Application of the Multiwell Productivity Index (MPI), SPE Journal 5, 1, 21-31, DOI: 10.2118/51793-PA.

Wattenbarger R.A., El-Banbi A.H., Villegas M.E., Maggard J.B. (1998) Production Analysis of Linear Flow Into Fractured Tight Gas Wells, SPE Paper 39931 presented at the SPE Rocky Mountain Regional/Low-Permeability Reservoirs Symposium, Denver, Colorado, USA, 5-8 April.

Yousef A.A., Jensen J.L., Lake L.W. (2009) Integrated Interpretation of Interwell Connectivity Using Injection and Production Fluctuations, Mathematical Geosciences 41, 1, 81-102, DOI: 10.1007/ s11004-008-9189-x.

Manuscript submitted in November 2014

Manuscript accepted in April 2015

Published online in September 2015

Cite this article as: M.S. Shahamat, H. Hamdi, L. Mattar and R. Aguilera (2016). A Novel Method for Performance Analysis of Compartmentalized Reservoirs, Oil Gas Sci. Technol 71, 38. 


\section{Appendix A}

Equations (9) and (10), which are the result of simple combination of material balance and deliverability equations construct a coupled system of two ordinary differential equations for two unknown $\left(p_{D M}\right.$ and $\left.p_{D M S}\right)$ since knowledge of $p_{D M}$ is required in order to find $p_{D M s}$ and vice versa. Using the notation that $X_{1}=p_{D M}$ and $X_{2}=p_{D M s}$, we can solve this system using matrix algebra, where the system is written in the following manner:

$$
X^{\prime}=A X+g
$$

In this equation, $X=\left[\begin{array}{l}X_{1} \\ X_{2}\end{array}\right], X^{\prime}=\frac{d X}{d t_{D M}}, A$ is the matrix of coefficients $A=\left[\begin{array}{cc}-F_{R} & F_{R} \\ F_{c} F_{R} & -F_{c} F_{R}\end{array}\right]$ and $g=\left[\begin{array}{l}1 \\ 0\end{array}\right]$.

As evident, this is a linear nonhomogeneous system of differential equations. In order to solve the above system and find the general solutions, we need to obtain the homogeneous solution (i.e. solution to the homogeneous system of ODE). This can be achieved by first evaluating the eigenvalues of Equation (A.1). The eigenvalues are obtained through finding the roots of the characteristic polynomial of the matrix of coefficients, i.e. $\operatorname{det}(A-\lambda I)=0$. Since $A$ in this case is a $2 \times 2$ matrix, then its characteristic polynomial is a second degree polynomial. Upon finding the roots, the following eigenvalues are obtained:

$$
\left\{\begin{array}{c}
\lambda_{1}=0 \\
\lambda_{2}=-F_{R}\left(1+F_{c}\right)
\end{array}\right.
$$

Then we need to find the eigenvectors $\vec{\alpha}=\left[\begin{array}{l}\alpha_{1} \\ \alpha_{2}\end{array}\right]$ and $\vec{\beta}=\left[\begin{array}{l}\beta_{1} \\ \beta_{2}\end{array}\right]$ corresponding to the first and second eigenvalues, respectively. These eigenvectors can be obtained according to the following equations:

$$
\begin{aligned}
& \vec{\alpha}=\left[\begin{array}{c}
1 \\
\frac{\lambda_{1}}{F_{R}}+1
\end{array}\right] \\
& \vec{\beta}=\left[\begin{array}{c}
1 \\
\frac{\lambda_{2}}{F_{R}}+1
\end{array}\right]
\end{aligned}
$$

After finding the eigenvectors, the homogeneous solution is obtained as below:

$$
X_{h}\left(t_{D M}\right)=C_{1} \vec{\alpha} e^{\lambda_{1} t_{D M}}+C_{2} \vec{\beta} e^{\lambda_{2} t_{D M}}
$$

Upon substitution and simplification, we obtain:

$$
X_{h}\left(t_{D M}\right)=C_{1}\left[\begin{array}{c}
1 \\
\frac{\lambda_{1}}{F_{R}}+1
\end{array}\right] e^{\lambda_{1} t_{D M}}+C_{2}\left[\begin{array}{c}
1 \\
\frac{\lambda_{2}}{F_{R}}+1
\end{array}\right] e^{\lambda_{2} t_{D M}}
$$

After finding the homogeneous solution, the particular solution can be obtained using the method of variation of parameters. For this purpose, we first construct the matrix $M$ using coefficients of $C_{1}$ and $C_{2}$ of the homogeneous solution (A.6):

$$
M=\left[\begin{array}{cc}
1 & e^{\lambda_{2} t_{D M}} \\
1 & \left(\frac{\lambda_{2}}{F_{R}}+1\right) e^{\lambda_{2} t_{D M}}
\end{array}\right]
$$

Here we substituted the first eigenvalue $\left(\lambda_{1}=0\right)$ to simplify the resulting matrix. Then we find the inverse of $M$ which upon simplification yields:

$$
M^{-1}=\frac{F_{R}}{\lambda_{2}}\left[\begin{array}{cc}
\left(\frac{\lambda_{2}}{F_{R}}+1\right) & -1 \\
-e^{-\lambda_{2} t_{D M}} & e^{-\lambda_{2} t_{D M}}
\end{array}\right]
$$


The particular solution is then obtained by finding the below integration:

$$
X_{p}\left(t_{D M}\right)=M \int M^{-1} g d t_{D M}
$$

The resulting particular solution is then determined to be according to below equation:

$$
X_{p}\left(t_{D M}\right)=\left[\begin{array}{c}
\left(\frac{\lambda_{2}+F_{R}}{\lambda_{2}}\right) t_{D M}+\frac{F_{R}}{\lambda_{2}{ }^{2}} \\
\left(\frac{\lambda_{2}+F_{R}}{\lambda_{2}}\right) t_{D M}+\frac{F_{R}}{\lambda_{2}{ }^{2}}\left(\frac{\lambda_{2}}{F_{R}}+1\right)
\end{array}\right]
$$

After finding the particular solution, the general solution is determined by adding the homogeneous and particular solutions:

$$
X_{g}\left(t_{D M}\right)=C_{1}\left[\begin{array}{l}
1 \\
1
\end{array}\right]+C_{2}\left[\begin{array}{c}
1 \\
\frac{\lambda_{2}}{F_{R}}+1
\end{array}\right] e^{\lambda_{2} t_{D M}}+\left[\begin{array}{c}
\left(\frac{\lambda_{2}+F_{R}}{\lambda_{2}}\right) t_{D M}+\frac{F_{R}}{\lambda_{2}{ }^{2}} \\
\left(\frac{\lambda_{2}+F_{R}}{\lambda_{2}}\right) t_{D M}+\frac{F_{R}}{\lambda_{2}{ }^{2}}\left(\frac{\lambda_{2}}{F_{R}}+1\right)
\end{array}\right]
$$

We can find the constants $C_{1}$ and $C_{2}$ using the initial conditions. We note here that the obtained results are for the average pressure. We also note that for the case of production at a constant rate and after reaching the boundaries of the first tank, the wellbore pressure tracks the average pressure. As a result we can set the initial condition as the starting wellbore pressure during $\mathrm{BDF}\left(p_{D M, B D F}\right.$, as obtained by the conventional methods of pressure transient analysis). Therefore the initial conditions are:

$$
X_{g}\left(t_{D M, B D F}\right)=\left[\begin{array}{c}
p_{D M, B D F} \\
0
\end{array}\right]
$$

Substitution of these initial conditions and simplification of the results give the following equation for $C_{1}$ and $C_{2}$ :

$$
\begin{gathered}
C_{1}=\left(\frac{\lambda_{2}+F_{R}}{\lambda_{2}}\right)\left(p_{D M, B D F}-t_{D M, B D F}\right) \\
C_{2}=\left(-\frac{F_{R}}{\lambda_{2}{ }^{2}}-\frac{F_{R}}{\lambda_{2}} p_{D M, B D F}\right) e^{-\lambda_{2} t_{D M, B D F}}
\end{gathered}
$$

Using these constants, the general solution $X_{g}\left(t_{D M}\right)$ is therefore obtained. The first element of the matrix $X_{g}\left(t_{D M}\right)$ is $p_{D M}$ which upon simplification yields:

$$
p_{D M}=p_{D M, B D F}+\left(1+\frac{F_{R}}{\lambda_{2}}\right)\left(t_{D M}-t_{D M, B D F}\right)+\frac{F_{R}}{\lambda_{2}}\left(\frac{1}{\lambda_{2}}+p_{D M, B D F}\right)\left(1-e^{\lambda_{2}\left(t_{D M}-t_{D M, B D F}\right)}\right)
$$

And the first element of the matrix $X_{g}\left(t_{D M}\right)$ is $p_{D M s}$ which is according to Equation (A.16):

$$
p_{D M s}=\left(1+\frac{F_{R}}{\lambda_{2}}\right)\left\{\left(t_{D M}-t_{D M, B D F}\right)+\left(\frac{1}{\lambda_{2}}+p_{D M, B D F}\right)\left\{1-e^{\lambda_{2}\left(t_{D M}-t_{D M, B D F}\right)}\right\}\right\}
$$

\section{Appendix B}

In this case, Equations (14) and (15), construct a coupled system of two ordinary differential equations for two unknown $\left(q_{D M}\right.$ and $q_{D M s}$ ), since knowledge of $q_{D M}$ is required in order to find $q_{D M s}$ and vice versa. Using the notation that $X_{1}=q_{D M}$ and $X_{2}=q_{D M s}$, we can solve this system using matrix algebra, where the system is written in the following manner:

$$
X^{\prime}=A X
$$


In this equation, $X=\left[\begin{array}{l}X_{1} \\ X_{2}\end{array}\right], \mathrm{X}^{\prime}=\frac{d X}{d t_{D M}}$ and $A$ is the matrix of coefficients given by $A=\left[\begin{array}{cc}-1 & 1 \\ F_{R} & -\left(F_{R}+F_{c} F_{R}\right)\end{array}\right]$.

This is a linear homogeneous system of differential equations. In order to solve the above system and find the general solutions, we need to obtain the homogeneous solution (i.e. solution to the homogeneous system of ODE). This can be achieved by first evaluating the eigenvalues of Equation (B.1). The eigenvalues are obtained through finding the roots of the characteristic polynomial of the matrix of coefficients, i.e. $\operatorname{det}(A-\lambda I)=0$. Since $A$ in this case is a $2 \times 2$ matrix, its characteristic polynomial is of second degree, as below equation shows:

$$
\lambda^{2}+\left(1+F_{R}\left(1+F_{c}\right)\right) \lambda-F_{R} F_{c}=0
$$

Depending on the discriminant for this equation, we can have real or imaginary roots. The discriminant for Equation (B.2) is as below:

$$
\Delta=\left(1+F_{R}\left(1+F_{c}\right)\right)^{2}-4 F_{R} F_{c}
$$

This equation can be manipulated to yield the following relation, which shows that we have a positive discriminant and therefore (B.3) has two real roots:

$$
\Delta=\left(1-F_{R}\left(1+F_{c}\right)\right)^{2}+4 F_{R}
$$

The roots are then obtained as below:

$$
\lambda=\frac{-\left(1+F_{R}\left(1+F_{c}\right)\right) \pm \sqrt{\left(1-F_{R}\left(1+F_{c}\right)\right)^{2}+4 F_{R}}}{2}
$$

We adopt the convention that $\lambda_{1}$ and $\lambda_{2}$ correspond to the positive (+) and negative (-) signs in Equation (B.5), respectively.

Then we need to find the eigenvectors $\vec{\alpha}=\left[\begin{array}{l}\alpha_{1} \\ \alpha_{2}\end{array}\right]$ and $\vec{\beta}=\left[\begin{array}{l}\beta_{1} \\ \beta_{2}\end{array}\right]$ corresponding to the first and second eigenvalues, respectively. These eigenvectors can be obtained according to the following equations:

$$
\begin{aligned}
& \vec{\alpha}=\left[\begin{array}{c}
1 \\
1+\lambda_{1}
\end{array}\right] \\
& \vec{\beta}=\left[\begin{array}{c}
1 \\
\lambda_{2}+1
\end{array}\right]
\end{aligned}
$$

Similar the previous case and after finding the eigenvectors, the homogeneous solution is obtained using Equation (A.5). Upon substitution and simplification, the homogeneous solution, which in this case is equal to the general solution gives:

$$
X_{g}\left(t_{D M}\right)=X_{h}\left(t_{D M}\right)=C_{1}\left[\begin{array}{c}
1 \\
\lambda_{1}+1
\end{array}\right] e^{\lambda_{1} t_{D M}}+C_{2}\left[\begin{array}{c}
1 \\
\lambda_{2}+1
\end{array}\right] e^{\lambda_{2} t_{D M}}
$$

We can find the constants $C_{1}$ and $C_{2}$ using the following initial conditions:

$$
X_{g}\left(t_{D M, B D F}\right)=\left[\begin{array}{c}
q_{D M, B D F} \\
0
\end{array}\right]
$$

Substitution of these initial conditions and simplification of the results give the following equation for $C_{1}$ and $C_{2}$ :

$$
C_{1}=\left(\frac{\lambda_{2}+1}{\lambda_{2}-\lambda_{1}}\right) q_{D M, B D F} \times e^{-\lambda_{1} t_{D M, B D F}}
$$




$$
C_{2}=-\left(\frac{\lambda_{1}+1}{\lambda_{2}-\lambda_{1}}\right) q_{D M, B D F} \times e^{-\lambda_{2} t_{D M, B D F}}
$$

Therefore from the obtained general solution we get the following equation for $q_{D M}$ :

$$
q_{D M}=q_{D M, B D F}\left\{\left(\frac{\lambda_{2}+1}{\lambda_{2}-\lambda_{1}}\right) \times e^{\lambda_{1}\left(t_{D M}-t_{D M, B D F}\right)}-\left(\frac{\lambda_{1}+1}{\lambda_{2}-\lambda_{1}}\right) \times e^{\lambda_{2}\left(t_{D M}-t_{D M, B D F}\right)}\right\}
$$

And for $q_{D M s}$ we obtain:

$$
q_{D M S}=q_{D M, B D F}\left(\frac{\left(\lambda_{1}+1\right)\left(\lambda_{2}+1\right)}{\left(\lambda_{2}-\lambda_{1}\right)}\right)\left\{e^{\lambda_{1}\left(t_{D M}-t_{D M, B D F}\right)}-e^{\lambda_{2}\left(t_{D M}-t_{D M, B D F}\right)}\right\}
$$

\section{Appendix C}

Dimensionless parameters used in well testing and production data analysis literature are as follows:

$$
\begin{gathered}
t_{D}=\frac{\beta_{2} k t}{\phi \mu c_{t}} \\
p_{D}=\frac{2 \pi k h}{\beta_{1} q \mu}\left(p_{i}-p_{w f}\right) \\
q_{D}=\frac{\beta_{1} q \mu}{2 \pi k h\left(p_{i}-p_{w f}\right)} \\
y_{e D}=\left(\frac{y_{e}}{x_{f}}\right)
\end{gathered}
$$

Using the definitions of capacitance and resistance for linear flow, we have:

$$
\begin{gathered}
c V=\left(\frac{4 x_{f} c_{t} h \phi}{5.615}\right) y_{e} \\
R=\left(\frac{\beta_{1} \mu}{k h}\right)\left(\frac{y_{e}}{x_{f}}\right)
\end{gathered}
$$

Using the above equations for capacitance and resistance in definition of $t_{D M}, p_{D M}$ and $q_{D M}$ (Eq. 5, 6 and 13) and upon simplification we obtain the following relation:

$$
\begin{gathered}
t_{D M}=\frac{t_{D}}{y_{e D}^{2}} \\
q_{D M}=q_{D} \times y_{e D}
\end{gathered}
$$




$$
p_{D M}=\frac{p_{D}}{y_{e D}}
$$

It has to be mentioned that in addition to the difference between $p_{D M}$ and $p_{D}$ shown in Equation (C.9), $p_{D M}$ is defined based on average pressure while the $p_{D}$ is in terms of the wellbore pressure. In addition, $q_{D M}$ is the linear equivalent of the dimensionless decline rate $\left(q_{D d}\right)$ defined by Fetkovich (1980). 